\title{
Photo-response of Two-Dimensional Ruddlesden-Popper Perovskite Films for Photovoltaics
}

\author{
Gao $\mathrm{Wu}^{1}$, Molang Cai ${ }^{1,2, *}$ and Songyuan Dai ${ }^{1,2, *}$ \\ ${ }^{1}$ State Key Laboratory of Alternate Electrical Power System with Renewable Energy Sources, North China Electric Power University, \\ Beijing 102206, P. R. China
}

${ }^{2}$ Beijing Key Laboratory of Novel Thin-Film Solar Cells, North China Electric Power University, Beijing, 102206, P. R. China

\begin{abstract}
Two-dimensional (2D) Ruddlesden-Popper (RP) perovskites have emerged as a prospective candidate to address the instability issues of traditional perovskite solar cells. However, the mechanisms of charge carrier transport of 2D perovskite films obtained by the solution process still remain elusive. In this work, we proposed a novel characterization technique based on the Kelvin probe force microscopy (KPFM) to investigate the micro-scale morphology and surface potential (SP) of the $\mathrm{BA}_{2} \mathrm{MA}_{3} \mathrm{~Pb}_{4} \mathrm{I}_{13}$ films. In additionally, a Xenon laser source was adopted to realize the in-situ scanning of the light response of the perovskite film. The obvious increase in surface potential values in the same scanning area before and after white light illumination indicated the emergence of photo-generated charge carriers. Based on the unique photophysical properties and form formation features of the hot-cast $\mathrm{BA}_{2} \mathrm{MA}_{3} \mathrm{~Pb}_{4} \mathrm{I}_{13}$ films, we fabricated the 2D perovskite solar cells (PSCs) with an efficiency of $10.95 \%$. As a result, the in-situ KPFM is capable to serve as an effective approach to investigating the charge carrier behaviors in the $2 \mathrm{D}$ perovskites for photovoltaic applications.
\end{abstract}

\section{Introduction}

To date, organic-inorganic hybrid perovskites (OIHPs) have emerged as a series of promising semiconductor materials ${ }^{[1-2]}$. Therefore, OIHPs have been extensively investigated for various optoelectronic applications, among which the rapidly evolved perovskite solar cells (PSCs) have drawn tremendous attention in photovoltaic (PV) research field, with the certificated power conversion efficiency (PCE) exceeding $25 \%{ }^{[3]}$. However, the commercialization of PSCs has been impeded by the instability issues when the devices are operating at realistic conditions ${ }^{[4]}$. Hence, researchers have proposed the two-dimensional (2D) perovskites as a promising candidate for obtaining highly stable $\mathrm{PSCs}^{[5]}$.

2D perovskites are derived from 3D perovskites that are generally described with the structural formula of $\mathrm{ABX}_{3}$, with insulating organic spacers tailoring the $3 \mathrm{D}$ perovskite octahedral framework into several (denoted as an integer $n$, hereinafter) blocks of separated slabs. Generally, when the bulky organic spacer (R) is monovalent, the type of $2 \mathrm{D}$ perovskites with the chemical formula of $\mathrm{R}_{2} \mathrm{~A}_{n-1} \mathrm{~B}_{n} \mathrm{X}_{3 n+1}$ is recognized as $2 \mathrm{D}$ Ruddlesden-Popper (RP) perovskites ${ }^{[6]}$. Owing to the intercalation of the hydrophobic bulky spacers, 2D perovskites exhibit distinct properties such as quantum confinement effects, high tunability and outstanding stability ${ }^{[7]}$. However, there are two major issues limiting the photovoltaic applications of 2D RP perovskites.
Firstly, due to the dielectric contrast of inorganic slabs and organic spacers, the exciton binding energy of 2D RP perovskites is relatively high, resulting in excitonic features and hinder the separation of electron-hole pairs ${ }^{\left[{ }^{[8]}\right.}$. Secondly, the insulating spacer cations and the fabrication crafts determine the self-assembly of 2D RP perovskite and always result in the multiple phase structure, leading to the multiple quantum-wells (MQWs) that greatly influence the charge carrier transport behaviors in the 2D perovskite solar cells (2D PSCs) ${ }^{[9-10]}$. Therefore, effective and intuitive characterization methods on the photo-response behaviors in the 2D RP perovskite films are urgently needed for further elucidating the mechanisms of the photo-induced charge carriers in the 2D PSCs.

In this work, we proposed a novel characterization technique based on the Kelvin probe force microscopy (KPFM) to realize the in-situ scanning of the morphology and surface potential (SP) profiles simultaneously. The in-situ KPFM techniques are able to detect the nanoscale morphology and electronic signals of the 2D RP perovskite film at high resolution without causing damages to samples. The emergence of the photo-generated charge carriers was successfully revealed from the remarkable increase in the SP values before and after the white light illumination. In addition, the typical film formation features of the $\mathrm{BA}_{2} \mathrm{MA}_{3} \mathrm{~Pb}_{4} \mathrm{I}_{13}$ films were investigated. Based on the characterization

*Corresponding author: molangcai@ncepu.edu.cn, sydai@ncepu.edu.cn 
results, we fabricated 2D PSCs with an efficiency of $10.95 \%$.

\section{Experimental}

\subsection{Film Preparation}

A hot-casting method was adopted to fabricate the 2D RP perovskite film and the photovoltaic device. The precursor solution of $\mathrm{BA}_{2} \mathrm{MA}_{3} \mathrm{~Pb}_{4} \mathrm{I}_{13}$ was prepared by dissolving BAI, MAI and $\mathrm{PbI}_{2}$ in the mixed solvents of DMF: DMSO (volume radio is 97:3) with the molar concentrations of $0.4,0.6$ and $0.8 \mathrm{~mol} / \mathrm{L}$ and the solution was stirred overnight in a nitrogen-purged glove box.

The patterned ITO-coated substrates $\left(15 \times 15 \mathrm{~mm}^{2}\right)$ were rinsed by sequential sonication for $15 \mathrm{~min}$ in each of the lotions including detergent, acetone, isopropanol, ethanol and deionized water. Afterwards, the clean substrates were dried under nitrogen flow and exposed to ozone plasma for $15 \mathrm{~min}$. For the hot-casting of the 2D RP perovskite film, the substrates were preheated on a hotplate at $60{ }^{\circ} \mathrm{C}$ for $10 \mathrm{~min}$. Then, the preheated substrate was quickly transferred to the spin coater and $80 \mu \mathrm{L}$ of the perovskite precursor solution was dropped to the substrates, followed by the spinning procedure at $4800 \mathrm{rpm}$ for $20 \mathrm{~s}$. After the spinning process, a thermal annealing of $100{ }^{\circ} \mathrm{C}$ for $10 \mathrm{~min}$ was processed to remove the solvent from the film. Notably, the spin coating was operated in the fume hood at the environmental temperature of $\sim 20^{\circ} \mathrm{C}$ and relative humidity of $\sim 25 \%$.

\subsection{Device Fabrication}

The 2D perovskite solar cells were fabricated based on an inverted planar configuration (glass/ITO/PTAA/ $\left.\mathrm{BA}_{2} \mathrm{MA}_{3} \mathrm{~Pb}_{4} \mathrm{I}_{13} / \mathrm{C} 60 / \mathrm{BCP} / \mathrm{Ag}\right)$. The ITO substrates were sequentially rinsed as described above. For the deposition of PTAA hole transport layer (HTL), $75 \mu \mathrm{L}$ of the PTAA/ chlorobenzene $(2 \mathrm{mg} / \mathrm{mL})$ solution were dripped to the substrates, followed by a spin coating process at $6000 \mathrm{rpm}$ for $30 \mathrm{~s}$. A thermal annealing of $100{ }^{\circ} \mathrm{C}$ for $10 \mathrm{~min}$ was processed to assist the crystallization of PTAA. The fabrication of $\mathrm{BA}_{2} \mathrm{MA}_{3} \mathrm{~Pb}_{4} \mathrm{I}_{13}$ active layer was hot-cast as mentioned above. The electron transport layer (ETL) of C60 and the BCP layer with the thicknesses of $30 \mathrm{~nm}$ and $9 \mathrm{~nm}$ respectively were successively deposited on top of the perovskite layer by thermal evaporation. Finally, $80 \mathrm{~nm}$ of $\mathrm{Ag}$ cathode was evaporated through masks and the fabrication of 2D PSCs was completed.

\subsection{Basic Characterizations}

A field-emission scanning electron microscopy (SEM, SU8010, Hitachi) was used to obtain the top-view morphology images of the $\mathrm{BA}_{2} \mathrm{MA}_{3} \mathrm{~Pb}_{4} \mathrm{I}_{13}$ film. The UVvis absorption curve of the film was measured with a spectrophotometer (UV-3600 Plus, Shimadzu). X-ray diffraction (XRD) pattern of the film was recorded by Xray diffractometer (Rigaku D max-2500) equipped with graphite copper $K \alpha$ radiation $(k=1.5418 \AA$ ) with the $2 \theta$ range from $5^{\circ}$ to $55^{\circ}$. The steady-state photoluminescence (PL) spectroscopy was conducted with an integrated spectral scanning test system (MStarter 100, Metatest Corporation).

For the photovoltaic performance of 2D PSCs, current density-voltage $(J-V)$ characteristics of the devices were measured by a solar simulator (Newport Class AAA) based on a Xenon lamp (150 W) and Keithley 2420 source under the radiation of 100 $\mathrm{mW} / \mathrm{cm}^{2}$ in the ambient environment. The active area of each cell was $0.043 \mathrm{~cm}^{2}$. The external quantum efficiency (EQE) curves of the device were tested using a spectral response test system (SOFN Instruments Co., Ltd.).

\subsection{The in-situ KPFM Measurements}

The in-situ KPFM platform was established on basis of the scanning probe microscopy system (Agilent Technologies 5500) coupled with a Xenon laser light source (LSH-X150, Zolix Instruments Co., Ltd). The topography and surface potential profiles of the 2D RP perovskite film were characterized simultaneously at the amplitude-modulation KPFM (AM-KPFM) mode and $\mathrm{Pt} / \mathrm{Ir}$-coated conductive tips (Nanosensors PPPEFM-20, $\sim 5 \mathrm{~N} / \mathrm{m}$ ) were used. The in-situ KPFM measurements were carried out on the same area without changing the sample under different illumination conditions, as depicted in Fig. 1. The profiling of topography and SP of the $\mathrm{BA}_{2} \mathrm{MA}_{3} \mathrm{~Pb}_{4} \mathrm{I}_{13}$ film was conducted within a range of $3 \times 3 \mu \mathrm{m}^{2}$, which presented the "dark-state" surface charge distribution of the $\mathrm{BA}_{2} \mathrm{MA}_{3} \mathrm{~Pb}_{4} \mathrm{I}_{13}$ film. Then, the Xenon laser light (8.48 A, light intensity: $\sim 200 \mathrm{~cd} / \mathrm{mm}^{2}$, outlet size: $0.5 \times 1.6 \mathrm{~mm}^{2}$ ) was continuously applied to the sample for $30 \mathrm{~s}$ and afterwards the scanning started again in the same area, which revealed the "lightinduced-state" surface charge distribution of the film ${ }^{[11]}$.

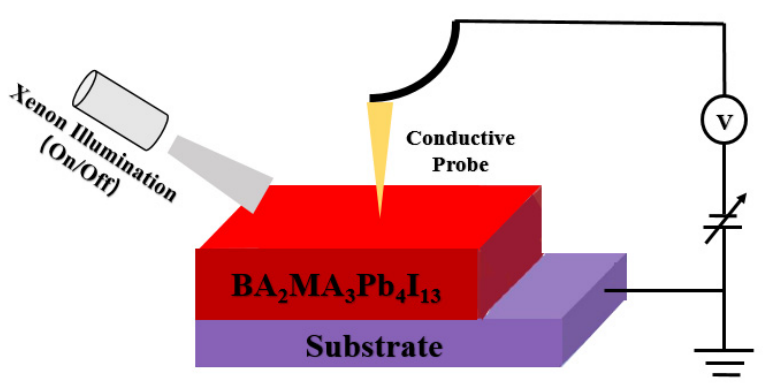

Figure 1. Schematic illustration of the in-situ KPFM measurements on the 2D RP perovskite film in the dark (Xenon light off) and under white light illumination (Xenon light on).

\section{Results and Discussion}

\subsection{Basic Film and Device Characterizations}

Fig. 2a showed the top-view SEM images of the $\mathrm{BA}_{2} \mathrm{MA}_{3} \mathrm{~Pb}_{4} \mathrm{I}_{13}$ film. The hot-cast film exhibited a uniform and compact surface morphology. Distinctive 
from the morphology of 3D perovskites consisting of grains with regular shapes ${ }^{[12]}$, it was difficult to observe grains and boundaries in the 2D RP perovskite film. In addition, there existed cracks in the surface of the film, which indicated the specific film formation features.

The UV-Vis absorption curve was depicted in Fig. 2 b. Exciton-like absorption peaks at $\sim 642 \mathrm{~nm}$ and $\sim 607 \mathrm{~nm}$ were observed, indicating the exsistence of the low $n$ value phases ( $n=4$ and 3, respectively) in the hot-cast $\mathrm{BA}_{2} \mathrm{MA}_{3} \mathrm{~Pb}_{4} \mathrm{I}_{13}$ film. Hence, the UV-Vis absorption curve clarified three dominant species in the 2D RP perovskite film from low $n$-value to high $n$-value ${ }^{[13-15]}$.

The X-ray diffraction (XRD) pattern of the $\mathrm{BA}_{2} \mathrm{MA}_{3} \mathrm{~Pb}_{4} \mathrm{I}_{13}$ film was shown in Fig. 2c. Three diffraction peaks at $14.08^{\circ}, 28.31^{\circ}$ and $44.74^{\circ}$ were well resolved, which matched the diffraction peak positions of $(\overline{1} 1 \overline{1}),(202)$ and (313) crystallographic planes from the 2D RP perovskites ${ }^{[12]}$. However, according to the XRD results on the $3 \mathrm{D}$ perovskite $\mathrm{MAPbI}_{3}$, the characteristic diffraction peaks were located at $14.05^{\circ}$, $28.45^{\circ}$ and $43.13^{\circ}{ }^{[13]}$, which was close to the results in Fig. 2c. We assumed that this might result from the dominant 3D-like phases $(n \rightarrow \infty)$ on top of the perovskite film.

The steady-state PL spectroscopy on the 2D RP perovskite film deposited on glass also confirmed the unique phase distribution phenomenon. As Fig. 2d showed, the PL spectrum was dominated by the emissions at 3D-like phases $(n \rightarrow \infty$, around $740 \mathrm{~nm})$, while no PL emmision peaks in the short wavelength region. This indicated that the $3 \mathrm{D}$-like species tended to accumulate on the top of the perovskite film. Through the hot-casting method, the vertical phase distribution with the low $n$-value (2D) and high $n$-value (3D-like) phases accumulated at the bottom and the top of perovskite film could facilitate the charge transfer across the device ${ }^{[15]}$.
The 2D PSCs based on the $\mathrm{BA}_{2} \mathrm{MA}_{3} \mathrm{~Pb}_{4} \mathrm{I}_{13}$ active layer was fabricated with the inverted planar structure of glass/ITO/PTAA/perovskite/C60/BCP/Ag, as shown in the inset of Fig. 2e. A reasonable PCE of $10.95 \%$ was recorded from current density-voltage $(J-V)$ scanning, as shown in Fig. 2e, with an open-circuit voltage $\left(V_{O C}\right)$ of $1.02 \mathrm{~V}$, short-circuit current density $\left(J_{S C}\right)$ of 15.35 $\mathrm{mA} / \mathrm{cm}^{2}$ and fill factor $(F F)$ of 0.70 (Table 1). The external quantum efficiency (EQE) and integrated current density $\left(J_{\text {int }}\right)$ of $14.93 \mathrm{~mA} / \mathrm{cm}^{2}$ were shown in Fig. 2f, well agreed with the measured $J_{S C}$ from $J-V$ scanning. Compared with other reports ${ }^{[16,17]}$, the $V_{O C}$ of 2D PSCs is generally high which exceeds $1.0 \mathrm{~V}$, while the $J_{S C}$ will be the key to further improving the PCE.

Table 1. A summary of photovoltaic parameters of 2D PSCs based on the hot -cast $\mathrm{BA}_{2} \mathrm{MA}_{3} \mathrm{~Pb}_{4} \mathrm{I}_{13}$.

\begin{tabular}{|c|c|c|c|c|c|}
\hline $\begin{array}{c}\text { Cell } \\
\text { Structure }\end{array}$ & $\begin{array}{c}\text { PCE } \\
(\%)\end{array}$ & $\begin{array}{c}V_{O C} \\
(\mathrm{~V})\end{array}$ & $\begin{array}{c}J_{S C} \\
\left(\mathrm{~mA} / \mathrm{cm}^{2}\right)\end{array}$ & $F F$ & $\begin{array}{c}J_{\text {int }} \\
\left(\mathrm{mA} / \mathrm{cm}^{2}\right)\end{array}$ \\
\hline $\begin{array}{c}\mathrm{p}-\mathrm{i}-\mathrm{n} \\
\text { inverted } \\
\text { structure }\end{array}$ & 10.95 & 1.02 & 15.35 & 0.70 & 14.93 \\
\hline
\end{tabular}

\section{2 in-situ KPFM Measurements on the Photo- Response of the 2D RP perovskite film}

Fig. 3 showed the topography images and surface potential (SP) profiles of the $\mathrm{BA}_{2} \mathrm{MA}_{3} \mathrm{~Pb}_{4} \mathrm{I}_{13}$ film in the dark state and under illumination condition. As Fig. 3a and $\mathrm{d}$ showed, the calculated root-mean-square (RMS) values of roughness of the $\mathrm{BA}_{2} \mathrm{MA}_{3} \mathrm{~Pb}_{4} \mathrm{I}_{13}$ film before and after Xenon laser illumination were $7.55 \mathrm{~nm}$ and $7.26 \mathrm{~nm}$, which was in accord with the SEM image (Fig. 2a) that exhibited a less undulating surface of the $2 D R P$ film. More importantly, the calculated RMS values were quite similar and the topography images were essentially identical, which proved that the scanning was conducted in the same position of the sample with high credibility. (a)

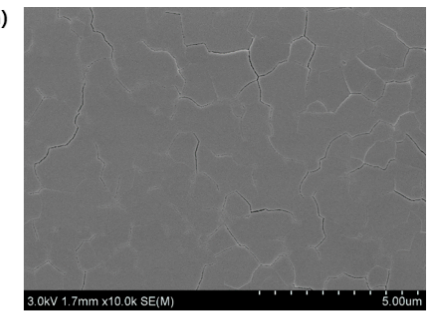

(d)

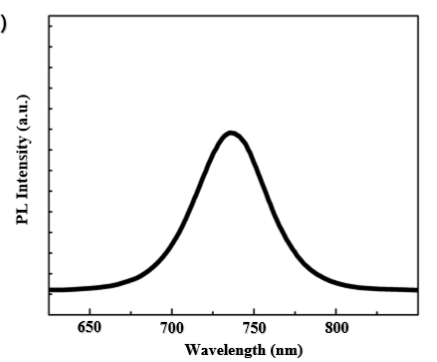

(b)
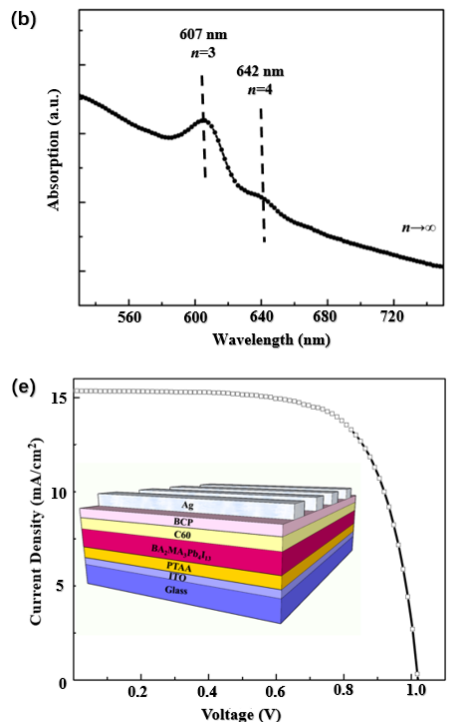
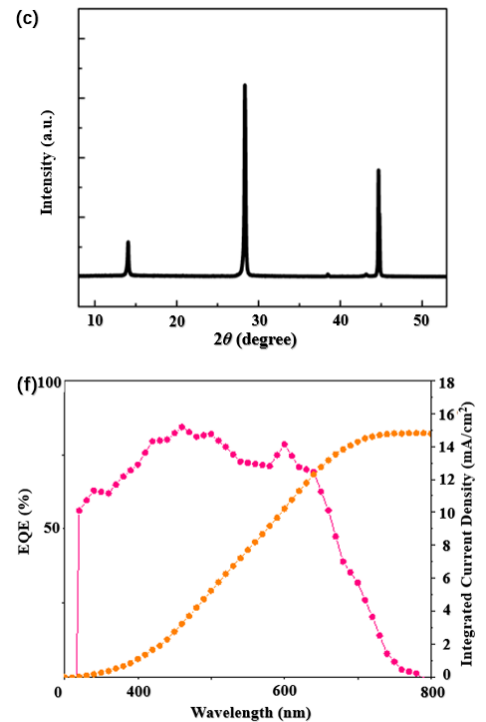

Figure 2. (a) Top-view SEM image, (b) XRD pattern, (c) UV-Vis absorption curve and (d) steady-state PL spectra of the $\mathrm{BA}_{2} \mathrm{MA}_{3} \mathrm{~Pb}_{4} \mathrm{I}_{13}$ film. The insets in Fig. 2d showed the incident $488 \mathrm{~nm}$ laser excited the top surface of the film. (e) $J-V$ curve and (f) EQE spectra of 2D PSCs based on the hot-cast $\mathrm{BA}_{2} \mathrm{MA}_{3} \mathrm{~Pb}_{4} \mathrm{I}_{13}$. The inset in Fig. 2e showed the inverted structure of the 2D PSCs. 
By unifying the scale bars of the two SP profiles (Fig. 3b and e), we found that the whole SP values of the $\mathrm{BA}_{2} \mathrm{MA}_{3} \mathrm{~Pb}_{4} \mathrm{I}_{13}$ film in the scanning regions improved dramatically after the illumination. This was ascribed to the generation of charge carriers when the perovskite film absorbed light. The photo-induced charge carriers would significantly influence the built-in electric field in the perovskite film ${ }^{[11]}$. Since the 2D RP perovskite film was deposited on glass, enormous electron-hole pairs generated might well be confined in the perovskite and then recombine due to the high exciton binding energy.

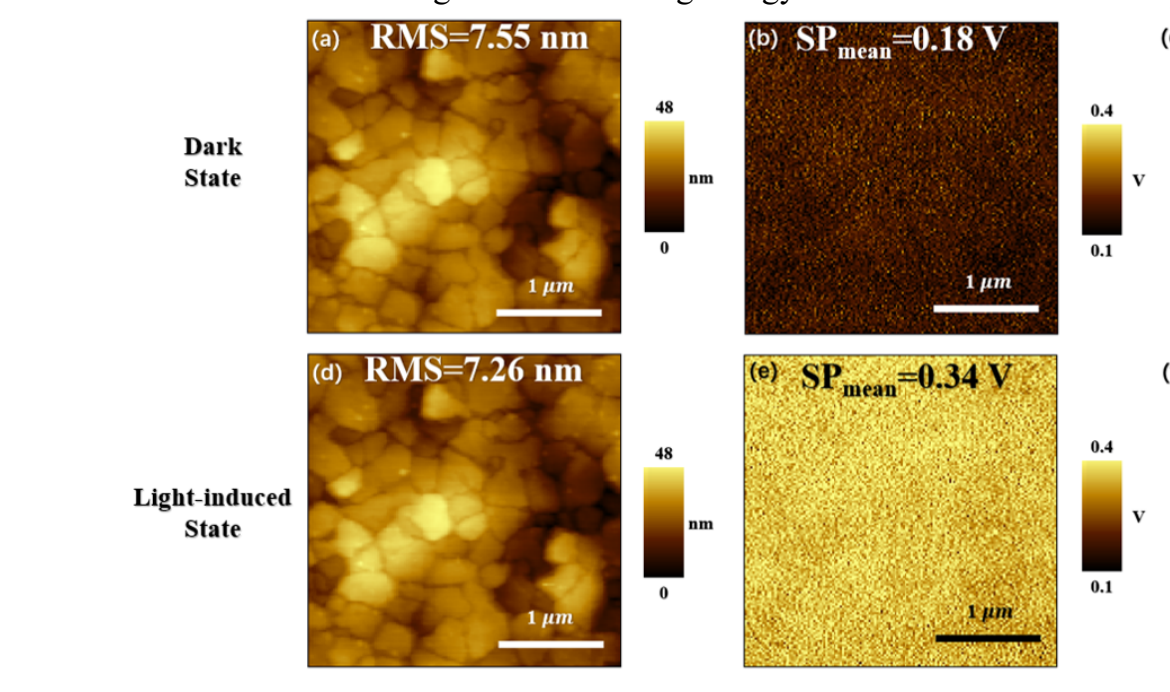

Notably, the histograms of SP distribution in the dark and light-induced states were depicted in Fig. 3c and $\mathrm{f}$. From the two histograms, it was confirmed that the overall SP values were improved after the Xenon laser excitation. The SP interval in the dark state was $\sim 0.05-0.27 \mathrm{~V}$, while that in the light-induced state was $\sim 0.21-0.48$ V. Also, the calculated mean surface potential $\left(\mathrm{SP}_{\text {mean }}\right)$ of the $\mathrm{BA}_{2} \mathrm{MA}_{3} \mathrm{~Pb}_{4} \mathrm{I}_{13}$ film increased dramatically from $0.18 \mathrm{~V}$ to $0.34 \mathrm{~V}$ after the Xenon laser illumination, in accord with the analyses above.
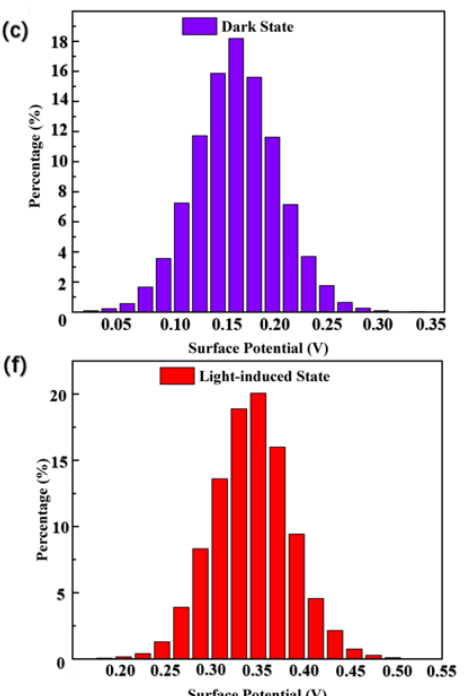

Figure 3. (a, d) Topography images and (b, e) surface potential profiles of the $\mathrm{BA}_{2} \mathrm{MA}_{3} \mathrm{~Pb}_{4} \mathrm{I}_{13}$ film in the dark state and light-induced state within the range of $3 \times 3 \mu \mathrm{m}^{2}$. (c, f) Histograms from the SP values of the $\mathrm{BA}_{2} \mathrm{MA}_{3} \mathrm{~Pb}_{4} \mathrm{I}_{13}$ film in the dark and light-induced states.

\section{Conclusion}

In this work, we proposed a novel characterization technique based on the Kelvin probe force microscopy (KPFM) coupled with a Xenon laser source to realize the in-situ scanning. The emergence of the photo-generated charge carriers was successfully revealed from the remarkable increase in the SP values in the same scanning area before and after the white light illumination. In addition, the typical film morphology and multiple phase distribution of the $\mathrm{BA}_{2} \mathrm{MA}_{3} \mathrm{~Pb}_{4} \mathrm{I}_{13}$ films were investigated. The fabricated 2D PSCs with an efficiency of $10.95 \%$. As a result, the establishment of in-situ KPFM platform paves the way to the research on the mechanisms of carrier behaviors in $2 \mathrm{D}$ perovskites.

\section{References}

1. F. Liu, J. Zhu, J. Wei, et al., Appl. Phys. Lett. 104, 253508 (2014).

2. G. Grancini, M. K. Nazeeruddin, Nat. Rev. Mater. 4, 4-22 (2019).

3. NREL, Best Research-Cell Efficiencies, (accessed: April 2021).

4. S. P. Dunfield, L. Bliss, F. Zhang, et al., Advanced Energy Materials, 10, 1904054 (2020).
5. Y. Chen, Y. Sun, J. Peng, et al., Advanced Materials, 30, 1703487 (2018).

6. D. Luo, R. Su, W. Zhang, et al., Nat. Rev. Mater. 5, 44-60 (2020).

7. B. Wang, J. Iocozzia, M. Zhang, et al., Chem. Soc. Rev. 48, 4854-4891 (2019).

8. L. He, Z. Xiao, X. Yang, et al., J. Mater. Sci. 55, 7691-7701 (2020).

9. S. Deng, E. Shi, L. Yuan, et al., Nat. Commun. 11, 664 (2020).

10. G. Wu, X. Li, J. Zhou, et al., Adv. Mater. 31, 1903889 (2019).

11. C. Lu, Z. Hu, Y. Wang, et al., Adv. Mater. Interfaces 6, 1801253 (2019).

12. H. Peng, M. Cai, J. Zhou, et al., Sol. RRL 4, 2000216 (2020).

13. Y. Lin, Y. Fang, J. Zhao, et al., Nat. Commun. 10, 1008 (2019).

14. G. Wu, T. Yang, X. Li, et al., Matter 4, 582-599 (2021).

15. T. He, S. Li, Y. Jiang, et al., Nat Commun 11, 1672 (2020).

16. M. S. de la Fuente, S. Kaur, Q. Hu, et al., ACS Energy Lett. 5, 109-116 (2020).

17. H. Tsai, W. Nie, J. C. Blancon, et al., Nature 536, 312-316 (2016). 